\title{
A survey on E-mail Security and Authentication Process
}

\author{
Namita Sahu \\ Department of \\ Computer Science \& Engineering \\ Lakshmi Narain \\ college of Technology, \\ Indore(M.P), India
}

\author{
Pawan Patidar \\ Department of \\ Computer Science \& Engineering \\ Lakshmi Narain \\ college of Technology, \\ Indore(M.P), India
}

\begin{abstract}
Online Email messages are not secured as they move crosswise over Internet. Messages can be undelivered or blocked and read by unapproved or unintended people. Email can likewise be surreptitiously altered even fashioned making the feeling that a individual created an impression that she didn't. Customary Internet email does not give methods to guaranteeing uprightness, security or making origin. A computerized signature or advanced mark plan is a sort of uneven cryptography used to the security properties of a transcribed mark on paper. Advanced steganography is the capacity to shroud data in an electronic source. We concentrate on novel information concealing methods gave by the field of steganography to confirm an encoded computerized signature, covered up in an advanced picture. There are no calculations existing as of now to secure email messages which utilize encryption what's more, picture steganography systems together. In this paper we talk about the usage of a calculation which utilizes these two procedures together and examine the execution of the framework. [1]
\end{abstract}

\section{Keywords}

Picture verification, One Time Password \& Key Security (PVOTPKS), Cryptography, Compression

\section{INTRODUCTION}

With fast improvements in correspondence advancements in view of PC and web, interchanges by means of messages has ended up more far reaching. On the other hand, customary email convention is frail since the message is transmitted in plain content. On the off chance that somebody needs to translate, duplicate or even adjust messages, they can do it without any difficulty. Singular securities for example, bank exchanges, business mysteries, even nations brainpower data are being conveyed through messages furthermore, along these lines substance of messages are presently more profitable than any other time in recent memory. Subsequently, the security of messages has raised more concerns. The safe informing framework has three advantages: keeping delicate data private, keeping anybody from messing around with the substance of the message and validating the character of both the message sender and collector.

It has wound up standard with the risky improvement of the web. Email accepts a crucial part in a human life. Email is comprehensively used inside far reaching scale affiliation; diverse e-exchange applications used the email for exchanging the information. The protection of email against diverse risk is exceptionally crucial so when a customer endeavour to log into a structure instead of simply affirming it through a fundamental substance watchword this work uses an additional picture level check. Right when an email get framed then it would get pressed by weight computation i.e. incident less weight estimation in which no loss of data happen after weight and decompression and after that mail will scramble by encryption count and after that encryption a unique key will make and that key will be one time pad key. This secured mail can simply access through that key and subsequently that key will send to at recipient adaptable number and when the gatherer need to scrutinize the email the beneficiary can open the email by that key here we are giving the thought of Best of Both the world by this approach the better level of affirmation can be given and the email security against the danger could be conceivable and alteration ambush, masking strike can in like manner be sidestepped. [2]

\subsection{Picture Security}

Picture based confirmation is fused to outfit additional security composed with OTP. With IBA, when the customer performs first time enrollment on a site, he settles on a choice of a couple secret classes of pictures that are definitely not hard to recall, for instance, pictures of trademark perspective, autos. Every time the customer logs in, a structure of discretionarily delivered pictures is displayed to the customer. The customer recognizes pictures that were at that point picked. One-time access code is made by the picked pictures, making the affirmation handle more secure than using simply a static substance watchword. It's inside and out less requesting and gainful for the customer because he needs to remember The above paper will focus on the email protection against the distinctive strike as we understand that email include two areas the header part and the body part when an email get made the .eml report get made that record is send to beneficiary end so first that archive will get stuffed by the weight estimation i.e. incident less weight in which no loss of data happen after weight and the decompression. After that an encryption will be completed by using one time pad and thereafter the key will be send at beneficiary flexible number by this an interloper cant get to the email content because the mail cant open until that key won't used with it Also when the gatherer get the key in his/her adaptable and need to get to the mail the deciphering can be performed by that astounding key [3] 


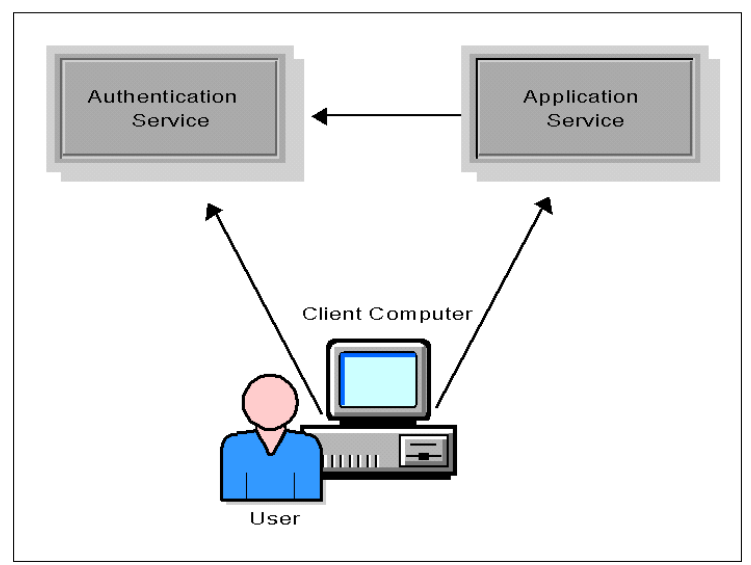

Fig:1 Picture Security

\subsection{Compression}

With a particular final objective to give better secure email transmission which is more secure against the distinctive ambush first the email weight is completed due to this weight by lossless weight count such a run length encoding by this the subtly, affirmation, get increases Example by using the run length encoding.

Run-length coding is a for the most part used and fundamental weight strategy which does not acknowledge a memory less source [4].

We supplant runs of pictures (maybe of length one) with sets of (run-length, picture)

- For test ,modestly clear sensible pictures, for instance, images line drawing and development

- It is greatly profitable for compacting bytes of a monochrome picture record, which routinely embodies a solid dim picture bits or "pixels", in a sea of white pixels, or the opposite it can in like manner be used sufficiently with shading outline records that contains gigantic clear bits.

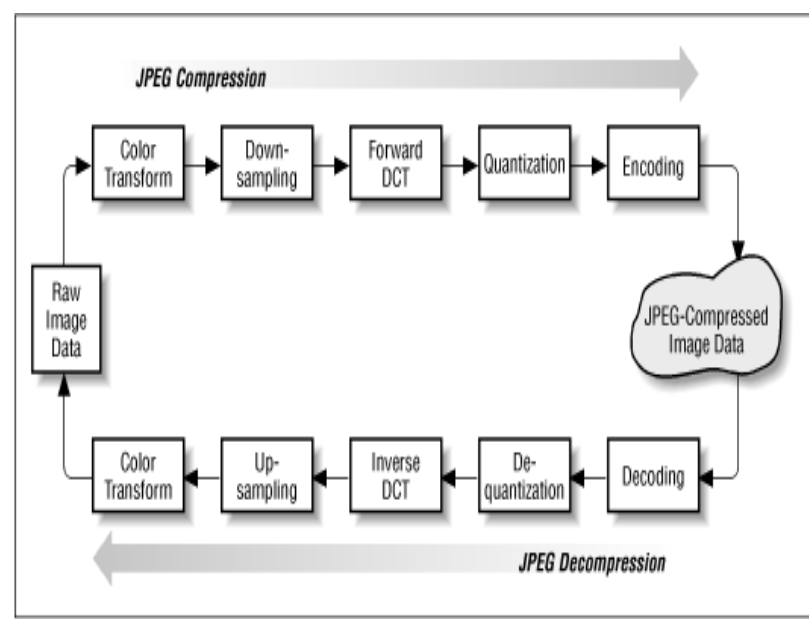

Fig:1 Compression Procedure

Email is a typical system for decision for the spread of infections and worms. Commonly, an infection will extricate email addresses in a tainted PC and send a duplicate of itself to some or these locations. These locations may be gotten from numerous sources, for example, the location book, attachment layer sniffing, inbox, sent envelope, and any of the put away email chronicles. Infection scanners can't stop an infection in its tracks unless the mark of the infection is known from the earlier. Lamentably, infection scholars have exhibited their persistent keenness by obstructing infection scanners with new infections that escape early location. Ceasing a polymorphic infection that uses a few purposes of passage can be an overwhelming errand utilizing conventional mark based infection filtering systems alone. Like spam email, numerous infections that are engendered through email today show different techniques to keep away from discovery by, receiving techniques, for example, changing the title,content body, and even appended document names and sort. This implies they will probably escape substance based sifting devices like infection scanners. Redesigning infection definitions in the future will probably be outdated with another era of infections that can transform their payload. To confuse this matter further, some infections look like innocuous spam, with no connections by any means. Rather, a client is coordinated to a webpage and may download hurtful executables without knowing so.[5]

\section{LITERATURE SURVEY}

In Amidst the most recent couple of years unmistakable examination articles had scattered which surrenders the inconspicuous parts to a certain level and in the wake of inspecting those some exceptional methodologies had been seen. Go on advances the study, underneath are some related works that partners this paper for further works.

In the paper [6], To secure against the abuse of email accounts through introduction of passwords, this paper propose that email reports be guaranteed using a customer specific email account interference revelation system. Not in any way like host or framework IDSs that are planned to guarantee one or more PCs, we acknowledge that an email record IDS should be proposed to secure one benefit: a customer's email storage facility. Reliably, an email narrative server then would truly be running different IDSs, with one case each customer. This layout choice is for the most part propelled by the amazingly singular nature of email; it similarly, on the other hand, has basic impact on our general system auxiliary arranging, exhibiting strategy, and the potential adaptability of the structure. More especially, the work on this issue with the going with danger model. In any case, expect that the attacker has permission to a customer's entire gear and programming environment: either the aggressor utilizes the same stage. As a beginning move towards building such a structure, developed a fundamental probabilistic model of customer email direct that join email senders and a customer's mentality of messages. In tests using data gathered from three months of viewed customer lead and designed models of aggressor direct, this model demonstrates a low rate of false positives (all around one false alarm every couple of weeks) while up 'til now getting by and large ambushes. These results suggest that inconsistency acknowledgment is a conceivable system for securing email archives, one that does not oblige changes in customer check or access conduct.

In the paper [7], The proposed novel programming security code encryption arrange in light of the rundown table. This technique uses a novel and beneficial encryption strategy called semi pack encryption for encryption the recorded table. It gives scarcest resemblance of the first data when encoded. Yet, semi cluster encryption is not compelling in diffusing the estimations of the plain substance. This disservice can be overcome by using changes. Hence, this system uses binded Hadamard changes and Number Theoretic Transforms to present scattering nearby the quasigroup change. The 
proposed technique is differentiated and the other encryption approaches and is seen to give better results.

In the paper [8], it gives a novel picture steganography procedure to hide messages or information inside other information in such a course as to not be distinguishable. This makes usage of the route that there is a great deal of data being traded reliably, making it hard to yield all the information for disguised messages. Customary cryptographic frameworks obscure the information, on the other hand it is still outstandingly pass that a message is being sent. Steganography tries to amend this deformity so an onlooker is not ready to know whether a message is being sent or not. This can be used as a piece of extension to standard cryptographic procedures, so the security may be updated, expecting that the routine frameworks are being used with the same meticulousness as sooner or later as of late. Steganography in pictures is each pixel is encoded as a movement of numbers which address the red green and blue qualities which make up the shading for that pixel. Since a slight change in this shading arrangement is not detectable by the human eye, it can be used to hide information. This is commonly satisfied by changing the smallest paramount bit, or LSB, for each pixel to identify with the bits of the covered message

The paper [9] proposed a novel The degree of the Proposal is obliged to the remote acceptance of trademark and legitimate components using electronic accreditations. For the reasons of this chronicle, we will consider remote approval to constitute an affirmation process where there is a certain physical division between the encouraging range of the application obliging check and the beginning stage of the character information on which the confirmation technique is based

\section{REVIEW OF LITERATURE AND PROBLEM}

statement

Heartbreakingly, the most usually used check accreditations, reusable passwords, are to an awesome degree exposed on account of fundamental samples of customer behavior. Various customers pick clear passwords that are definitely not hard to remember; various such passwords, of course, can be bartered by online and disengaged from the net word reference attacks. Customers enter passwords on untrusted machines that may be polluted with diseases, spyware, or diverse malicious programming. Such malware can be used to catch passwords. Moreover, customers consistently grant passwords across over spaces and applications, allowing one weak application (e.g. one that sends passwords free) to result in the exchange off other, more secure structures. Moreover, customers routinely reveal passwords to mates, relatives, and teammates. Now and again unexpectedly, yet here and there to energize the bestowing of information or resources. Those to a great degree same insiders regardless, regularly have method of reasoning in bartering a customer's security. [10]

Also by inspecting the predetermined composition \& other material a rate of the orchestrated issues or issues are recognized which is reliably exchanging off the security. These issues need to be overcome to give a better customer satisfaction in respects than the safe email archives. These are:

- User records in Emails are not secure.

- Users have no impact over security

- Dependency on more prepared substance based acceptance
- Attack related to channel can without a doubt decipher the messages

- Single instrument is not sufficient for security

- Compression must be lossless to be used mind

While considering the previously stated issues this work proposes a novel multilevel email security building configuration. This security development demonstrating is in light of three essential phrasings. These are Image Authentication, Compression \& Cryptography.

\section{CONCLUSION AND FUTURE WORK}

As we realize that email security is a critical issue and numerous programming based arrangements were created to give email security yet they were sufficiently bad to give security. So this work proposes the new idea of the multilevel email files security structural engineering ISA-CC through known parameters. Improved functionalities like picture validation, pressure by lossless pressure calculation and encryption utilizing AES, DES with one time cushion which can be better answer for give security and it can evade different assaults over email. It may be different calculations for securing recreating the data from the target picture yet the greater part of them continue from some measure of disappointment of emit information while remaking it.The proposed technique may be utilized to attain to all the principle objectives of cryptography by a solitary mean. IACOTPC comprises of extremely straightforward steps with no rounds when contrasted with the standard hash and MAC calculations. It would doubtlessly have low overhead, so the target of accessibility would be attained to. Encryption is finished with the most recent secure encryption standard AES, so Confidentiality is guaranteed.[11]

\section{REFERENCES}

[1] Soheb Munir, A.S.Zadgaonkar and Manish Shrivastava "Key Generation and Verification for Image Authentication", International Journal of Advanced Computer Research (ISSN (print): 2249-7277 ISSN (online): 2277-7970) Volume-3 Number-3 Issue-12 September-2013

[2] Suresh Kumar B. and Jagathy Raj V. P. "A Secure Email System Based on IBE, DNS and Proxy Service" Journal of Emerging Trends in Computing and Information Sciences@2009-2012 CIS Journal.

[3] Abhas Tandon,Rahul Sharma, Sankalp Sodhiya and P.M.Durai Raj Vincent "QR Code based secure OTP distribution scheme for Authentication in Net-Banking" in International Journal of Engineering and Technology (IJET). Vol 5 No 3 Jun-Jul 2013

[4] Shabir Ahmad and Bilal Ehsan " The Cloud Computing Security Secure User Authentication Technique (Multi Level Authentication)." International Journal of Scientific \& Engineering Research, Volume 4, Issue 12, December-2013 2166 ISSN 2229-5518

[5] Ms.B.Veera Jyothi, Dr.S.M.Verma and Dr.C.Uma Shanker "Implementation and Analysis of Email Messages Encryption and Image Steganography Schemes for Image Authentication and Verification" in IJCA Auguest-2014

[6] Neeta Wadhwa, Syed Zeeshan Hussain and S.A.M Rizvi "A Combined Method for Confidentiality, Integrity, 
International Journal of Computer Applications (0975 - 8887)

Volume 154 - No.3, November 2016

Availability and Authentication (CMCIAA)" WCE 2013, July 3 - 5, 2013, London, U.K.

[7] Suresh Kumar B. and Jagathy Raj V. P. "A Secure Email System Based on Identity Based Encryption" IJWCNT Volume 1, No.1, August- September 2012

[8] Shreya Zarkar, Sayali Vaidya, Achal Bharambe, Arifa Tadvi and Tanashree Chavan "Secure Server Verification by using Encryption Algorithm and Visual Cryptography” IJSR Volume 3 Issue 12, December 2014

[9] Yiru Li and Anil Somayaji "Securing Email Archives through User Modeling "School of Computer Science,
Carleton University1125 Colonel By Drive, Ottawa, ON K1S 5B6 Canada

[10] Sasirekha N and Hemalatha M , "An Enhanced Code Encryption Approach with HNT Transformations for Software Security", International Journal of Computer Applications (0975 - 8887) Volume 53- No.10, September 2012

[11] Salvatore J. Stolfo, Chia-Wei Hu, Wei-Jen Li,Shlomo Hershkop and Ke Wang, Olivier Nimeskern "Combining Behavior Models to Secure Email Systems" DARPA contract F30602-00-1-0603 